\title{
Metabolic Modeling and Simulation Analysis of Thyroid Disorder Pathway
} Manish Kumar Gupta ${ }^{1}$, Dev Bukhsh Singh ${ }^{2}$, S. K. Rath ${ }^{3}$ and Krishna Misra ${ }^{4 *}$

${ }^{1}$ Department of Bioinformatics, University Institute of Engineering and Technology, Chhatrapati Shahu Ji Maharaj University, Kanpur, India ${ }^{2}$ Department of Biotechnology, Institute of Biosciences and Biotechnology, Chhatrapati Shahu Ji Maharaj University, Kanpur, India ${ }^{3}$ Toxicology Division, Central Drug Research Institute, Lucknow - 226001, India

${ }^{4}$ Centre of Biomedical Magnetic Resonance, Sanjay Gandhi Post-Graduate Institute of Medical Sciences Campus Raebareli Road, Lucknow - 226 014, India

\begin{abstract}
Thyroid hormone secretion pathway is one of the important pathways that regulates growth, development and is considered critical for brain, skeletal development and maturation. Autoimmune Thyroid Disease (AITD) results in damage of the thyroid gland altering the normal secretion of thyroid hormones causing hypothyroidism (Hashimoto's thyroiditis) or hyperthyroidism (graves' disease). A map of molecular interaction of the thyroid stimulating hormone receptor has been created using systems biology graphical notation language with the help of CellDesigner 4.1 and converted into BioPax 2.8.2 pathways description format. In the current state the map contains 9 compartment, 32 simple and complex protein, 18 small molecules, 3 ions and 35 chemical reactions. The network contains more details about Thyroid hormones, Thyroxin (T4) and Triiodothyronin (T3), secretion pathway than existing large scale real pathways. Simulation was done in order to understand the time-dependent behavior of TSH, T3 and T4 by taking 16 different cases related thyroid disorder. The simulation patterns are invariable after passing with certain period, it does not deviate the simulation pattern of pathways. This study helps in identification of novel targets related with different types of thyroid disorder. To anticipate potential drug targets by system-wide analysis of the metabolic network for the effective treatment of thyroid disorder, the model can be useful.
\end{abstract}

Keywords: Metabolic modeling; Simulation; Thyroid stimulating hormone receptor; Graves' disease; Hashimoto's thyroiditis

\section{Introduction}

Autoimmune thyroid disease (AITD) is a common organ specific autoimmune disorder affecting thyroid gland mostly in women [1]. Thyroid gland is affected by autoimmunity in which the immune system which is "self-defense mechanism" attacks and damages the thyroid of an individual. This autoimmunity to thyroid is the result of interaction of many genetic and environmental factors which renders Thyroid Stimulating Antibodies (TSAbs) and Thyroid Blocking Antibodies (TBABs) to compete with Thyroid Stimulating Hormone (TSH) for binding with thyroid stimulating hormone receptor and do not let the TSH to bind with its receptor resulting in hyper or hyposecretion of thyroid hormones $[2,3]$. The thyrotropin receptor (TSHR) is the target of the immune system in Graves' disease, and auto antibodies directed against this antigen are the direct cause of clinical hyperthyroidism. The difference between a TSAb and a TBAb on the molecular level is still unsolved [4].

The diagnosis and therapy of Graves' disease required better understanding of molecular interaction between TSHR auto antibodies and the TSHR [5].Various information related to thyroid disorders were retrieved from different literature for the simulation of thyroid model [6-10].

All biological systems exhibit complexity which is an important to be well understood for any research. To solve this problem, Pathway modeling approach has come into existence which understands and analyzes the complex reactions. This approach can further help in designing new drugs for autoimmune diseases [11]. Such an attempt has been made in the present work.

The goal of Systems biology is analysis of a disease at molecular level considering the whole organism which helps in understanding the physiology of disease to build a regulatory and metabolic pathway [12,
13]. Today pathway modeling has become an important approach to study the complex reactions which take place in a system and also to understand the alternations that take place in normal pathway which results in diseased state. The metabolic modeling is more important than signaling pathways due to its direct thermodynamics and special stoichiometric constraints [14].

In order to make the map a realistic and available resource, it must be available in a standard language format. Hence, the map can understand in machine readable language i.e. Systems Biology Markup Language (SBML) [15]. It accepts a clear graphical notation design called the process diagram, which aims to present a standard for demonstrating molecular communications in an explicit way [16].

In this study the approach of pathway modeling has been applied to understand normal secretion of thyroid hormones by designing the normal metabolic pathway of T3 and T4 secretion. Over secretion of thyroid hormones in explained by designing the pathway depicting the role of Thyroid Stimulating Antibodies (TSAbs) and under secretion of thyroid hormones by designing the pathway showing the role of Thyroid Blocking Antibodies (TBABs).

\section{Materials and Methods}

An exhaustive literature search of different paper based on auto

*Corresponding author: Krishna Misra, Centre of Biomedical Magnetic Resonance, Sanjay Gandhi Post-Graduate Institute of Medical Sciences Campus, Raebareli Road, Lucknow - 226 014, India, Tel: +91 9415247579, E-mail: krishnamisra@hotmail.com

Received Febraury 16, 2012; Accepted April 27, 2012; Published April 30, 2012

Citation: Manish Kumar G, Dev Bukhsh S, Rath SK, Krishna M (2012) Metabolic Modeling and Simulation Analysis of Thyroid Disorder Pathway. J Comput Sci Syst Biol 5: 052-061. doi:10.4172/jcsb.1000090

Copyright: () 2012 Manish Kumar G, et al. This is an open-access article distributed under the terms of the Creative Commons Attribution License,which permits unrestricted use, distribution, and reproduction in any medium, provided the original author and source are credited. 
Citation: Manish Kumar G, Dev Bukhsh S, Rath SK, Krishna M (2012) Metabolic Modeling and Simulation Analysis of Thyroid Disorder Pathway. J Comput Sci Syst Biol 5: 052-061. doi:10.4172/jcsb.1000090

immune thyroid disorder (graves' and hashimoto's disease) was done in order to identify simple and complex proteins, molecules and ions.

\section{Notation used in the model}

The major symbols used to characterize molecules and interactions in model preparation are shown in Figure 1. A graphical notation system for biological networks to express enough information in a clearly visible and explicit way was used [17].

In the general organization of the diagram, a total of 9 compartments, 32 simple and complex proteins, 18 small molecules and 3 ions have been used for making Thyroid Hormone Secretion model. Besides that 35 reactions are involved in thyroid hormone secretion pathways. It compiles experimental results from more than seventy publications. Once the model was built in CellDesigner software, the pathway information was exported into BioPAX format.

\section{Biological databases}

The Kyoto Encyclopedia of Genes and Genomes (KEGG) pathway database section was used for retrieving of AITD pathways (http:// www.genome.jp/kegg/pathway/hsa/hsa05320.html), is a compilation of manually drawn pathway maps representing our familiarity on the molecular interaction and reaction network. The AITD pathway is having entry hsa05320. Thyrotropin-releasing hormone receptor signaling pathway was retrieved from panther database (http://www. pantherdb.org/pathway/pathwayList.jsp) and merged with thyroid hormone secretion model. PANTHER (Protein Analysis through Evolutionary Relationships) Classification System is an exclusive source that categorizes genes by their functions, using published scientific experimental proof and evolutionary relationships to predict function even in the absence of direct experimental evidence [18]. Pubmed was used to search various Research paper related to thyroid disorder pathways (http://www.ncbi.nlm.nih.gov/pubmed/).

\section{Software}

Cell designer 4.1: The Thyroid hormone model of Normal Hormone Secretion, Hyperthyroidism and Hypothyroidism were built with CellDesigner ver 4.1 and stored in System biology Markup Language (SBML) format. Acquiescence of CellDesigner with SBML enables us to store models and to exercise them for analyses by Other SBML-compliant applications. CellDesigner can incorporate with the entire SBW-enabled modules, together with simulation and other analysis packages. The latest version link directly with SGB, DBGET, iHOP, Genome Network Platform Pubmed, EntrezGene, MetaCyc, GeneWiki. Already built model can be imported from PANTHER database and Biomodels. CellDesigner can thus be a portal software stage and information manager for systems biology study. The Simulation was performed with SBML ODE Solver Library (SOSlib).
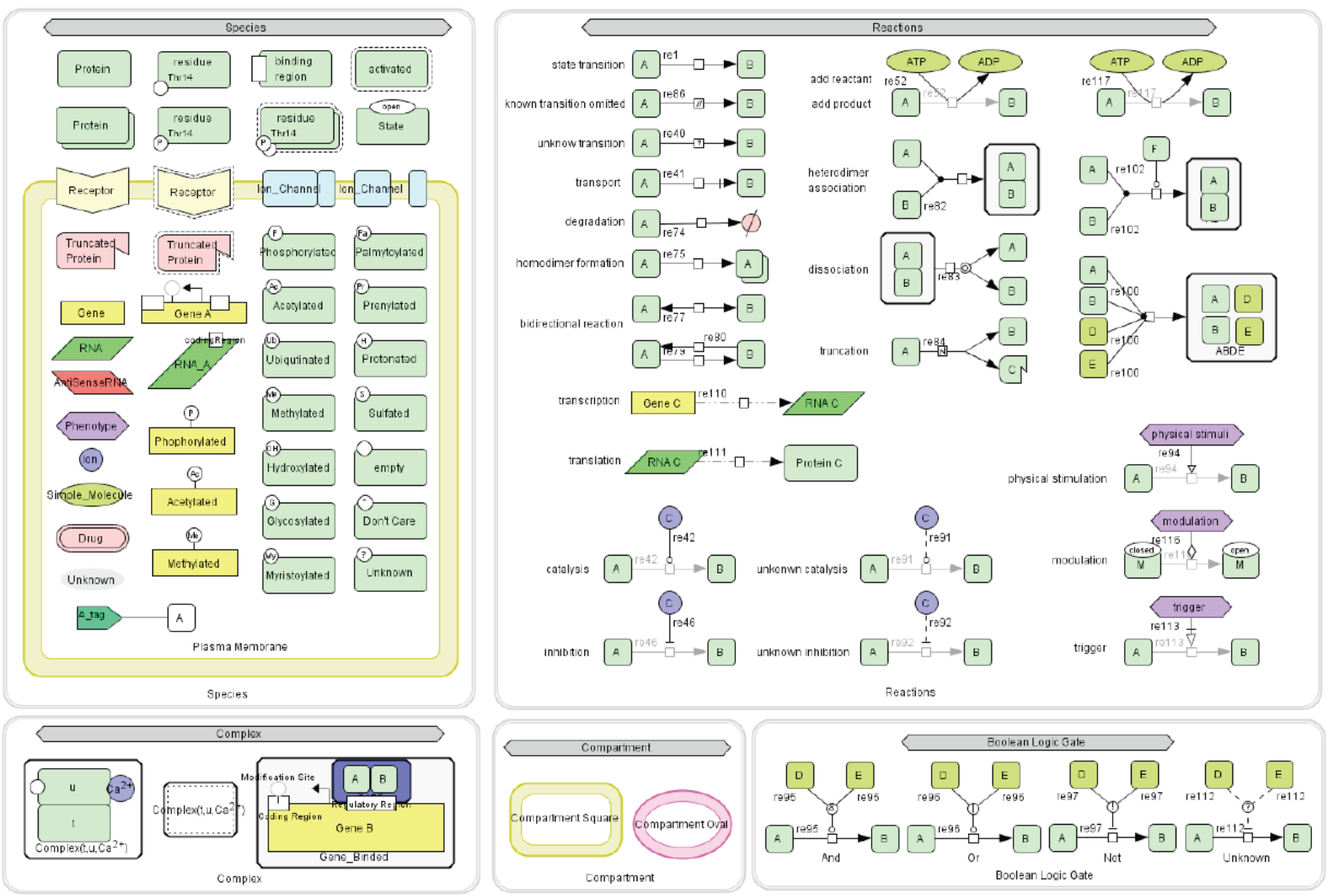

Figure 1: Symbols are provided in CellDesigner 4.1 for Species, Reaction, Complex Compartment and Boolean Logic Gate. 
SOSlib is both a programming library and a command-line purpose for symbolic and numerical study of a system of Ordinary Differential Equations (ODEs) derived from a chemical reaction network encoded in the SBML [19-22].

SBML squeezer 1.3: SBMLsqueezer 1.3 is a plug in used in CellDesigner to generate kinetic equations for biochemical pathways according to context of each reaction. When used as a plug-in for CellDesigner it uses the information from the SBGN representation of all network components. SBMLsqueezer assess the Systems Biology Ontology (SBO) annotations to pull out this information. The rate laws that can be created by SBMLsqueezer comprise numerous types of generalized mass action; comprehensive and generalized enzyme kinetics, several types of Hill equations, $\mathrm{S}$ - and $\mathrm{H}$-systems, along with additive models for gene regulation. User defined settings denote which equation to be relevant for any sort of reaction and how to make sure unit reliability of the model [23].

Cytoscape 2.8.2: Thyroid hormone model generated in CellDesigner was exported in BioPax format, which was imported in the Cytoscape with BiNoM plug in. BiNoM is a Cytoscape plug in [24], to assist the operation on biological networks represented in standard systems biology formats (SBML, SBGN, BioPAX) and to carry out studies on the network Structure. SBGN is Standard representation of essential biochemical and cellular processes with the help Set of symbols, Semantics and Syntax. The modular view of the Normal Thyroid hormone secretion, Hyperthyroidism and Hypothyroidism model were saved. Cytoscape 2.8.2 is an open source software platform for network visualization and biological data integration. A lot of plug in are available for various kinds of problem domains, including Cheminformatics, network analysis, Network Modification tools and semantic web [25-28].

\section{Results and Discussion}

The goal of this study is to exercise the dynamic behaviour of the thyroid hormone secretion pathway in the model by evaluating the biological experiment. The diagram utilizes SBGN system to symbolize proteins and their precise alteration, protein complexes as well as different protein transformation (state transition, dissociation, transport and so on) and their consequence of activation or inhibition of chemical reactions, together with transcriptional activation or inhibition.

A study has shown that TSH binding based on TSHR sequences recognized by the antibodies and suggested 3 distinct TSH binding regions in the TSHR: aa 246-260, 277-296, and 381-385. These regions may fold together to form a complex TSH-binding pocket $[29,30]$. Figure 2 (a) depicts the normal secretion of thyroid hormones in the body. When the blood temperature becomes too low, then a negative feedback mechanism takes place to increase production of Thyrotropin Releasing Hormone (TRH) which is transported by portal vein to anterior pituitary and secretes TSH. TSH binds with TSHR and results in activation of G-protein, which is composed of 3 subunits, G-alpha, G-beta and G-gamma. The G-alpha subunit dissociates and binds to Adenylate Cyclase (AC), which in turn gets activated and converts ATP to CAMP. This CAMP now binds to regulatory subunit of Protein Kinase ( $\mathrm{PKa}$ ) and gets phosphorylated which lead to activation of transcription factors like sodium iodide symporter (NIS) so that synthesis of thyroglobulin ( $\mathrm{Tg}$ ) can takes place[31-33]. Thereafter, iodide is transported to thyroid follicular cell in exchange for sodium via sodium iodide symporter (NIS), which then reacts with hydrogen peroxide $\left(\mathrm{H}_{2} \mathrm{O}_{2}\right)$ and converted into active iodine. This reaction is catalyzed by Thyroid Peroxidase(TPO). Active iodine combines with the tyrosine molecule present in $\mathrm{Tg}$ and iodinates these tyrosine residues which results in formation of Mono-iodothyronine (MIT) and Di-iodothyronine (DIT). Finally, coupling reaction takes place between MIT - DIT and DIT - DIT resulting in the formation of T3 and T4 which are catalyzed by TPO. Thyroid epithelial cells swallow colloid by endocytosis which contains $\mathrm{Tg}$. These endosomes are now fused with lysosomes which contain hydrolytic enzymes that digest $\mathrm{Tg}$ and liberate free thyroid hormones. Now, the free thyroid hormones diffuse out of lysosome and are transported to blood [32,34]. Figure 2(b) was obtained by SBW auto layout (Ver 0.7). It is the Randomized view of the Normal metabolic pathway of thyroid hormone secretion.
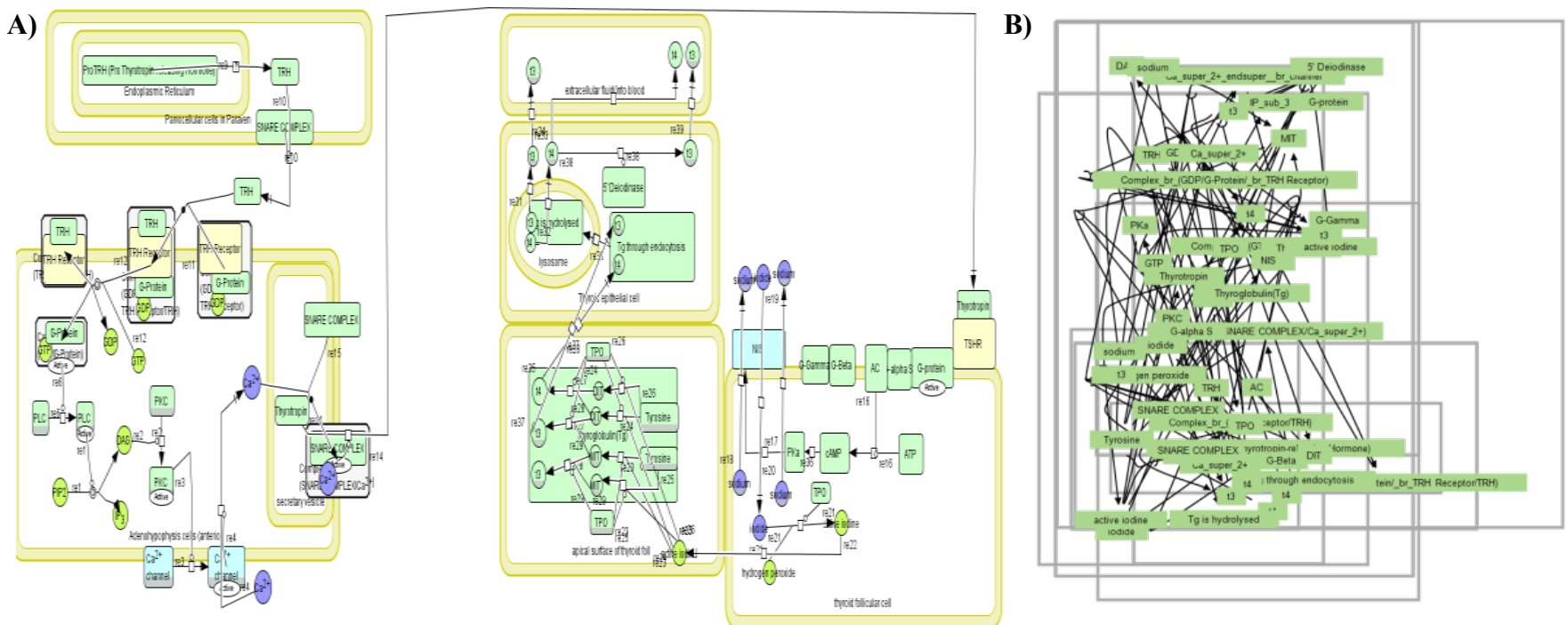

Figure 2: (a) Normal metabolic pathway of thyroid hormone secretion (SBGN View) 2(b) SBW autolayout (Ver 0.7) General Network Lay out view. 
Citation: Manish Kumar G, Dev Bukhsh S, Rath SK, Krishna M (2012) Metabolic Modeling and Simulation Analysis of Thyroid Disorder Pathway. J Comput Sci Syst Biol 5: 052-061. doi:10.4172/jcsb.1000090

The metabolic model of hyperthyroidism is shown in Figure 3(a), where thyroid stimulating antibodies (TSAb) play an important role for stimulation of T3 and T4. These antibodies bind themselves with TSHR instead of TSH and mimic the action of TSH. Due to this there is continuous secretion of thyroid hormones and the concentration of TSH becomes very less than its normal concentration. The pathway model of hypothyroidism has been categorized into Atrophic thyroiditis and Hashimoto thyroiditis. In case of Atrophic thyroiditis, thyroid blocking antibodies (TBAb) bind with TSHR and do not let the TSH to bind with TSHR [35]. These antibodies block all the reactions performed by TSH, therefore, secretion of thyroid hormones cannot take place. So, the concentration of TSH becomes more than the normal concentration and it is continuously secreted from TRH but cannot bind with TSH and the concentrations of thyroid hormones become less than that of the normal concentration as there is no secretion of thyroid hormones. Hashimoto thyroiditis is differentiated by the existence of goitre, thyroid autoantibodies against TPO and Tg in serum and variable level of thyroid dysfunction. Disease progression leads to the apoptosis of thyroid cells. Both autoantibodies and thyroidspecific Cytotoxic T Lymphocytes (CTLs) have been proposed to be responsible for autoimmune thyrocyte depletion. In case Hashimoto thyroiditis TPO is not able to catalyzed the conversion of MIT and DIT to T3 and T4 hormone as a result of mutation [36].

\section{Modular representation of the pathway}

SBML model was exported in BioPax format and imported in Cytoscape 2.8.2 and pathway saved in modular view. Identification of these modules allowed us to compile a modular hyperthyroidism pathway view. Network modules reveal that the 108 nodes are connected by 104 edges including 14 connected components and representing 'activation' and 'inhibition' relations. The information about these relations is derived from the detailed diagram (Figure 4).

In this work, an illustration of the molecular interactions regulating thyroid hormone secretion by pituitary and thyroid gland are presented. A significant amount of process data with both a thorough and a brief and readable representation were integrated. This map imitates our understanding of the several publications used to construct the metabolic pathway. This study helps us understanding the functioning of thyroid hormone secretion pathway and for integrating this information into realistic computational models. In some particular pathways biological publications often include indistinct statement
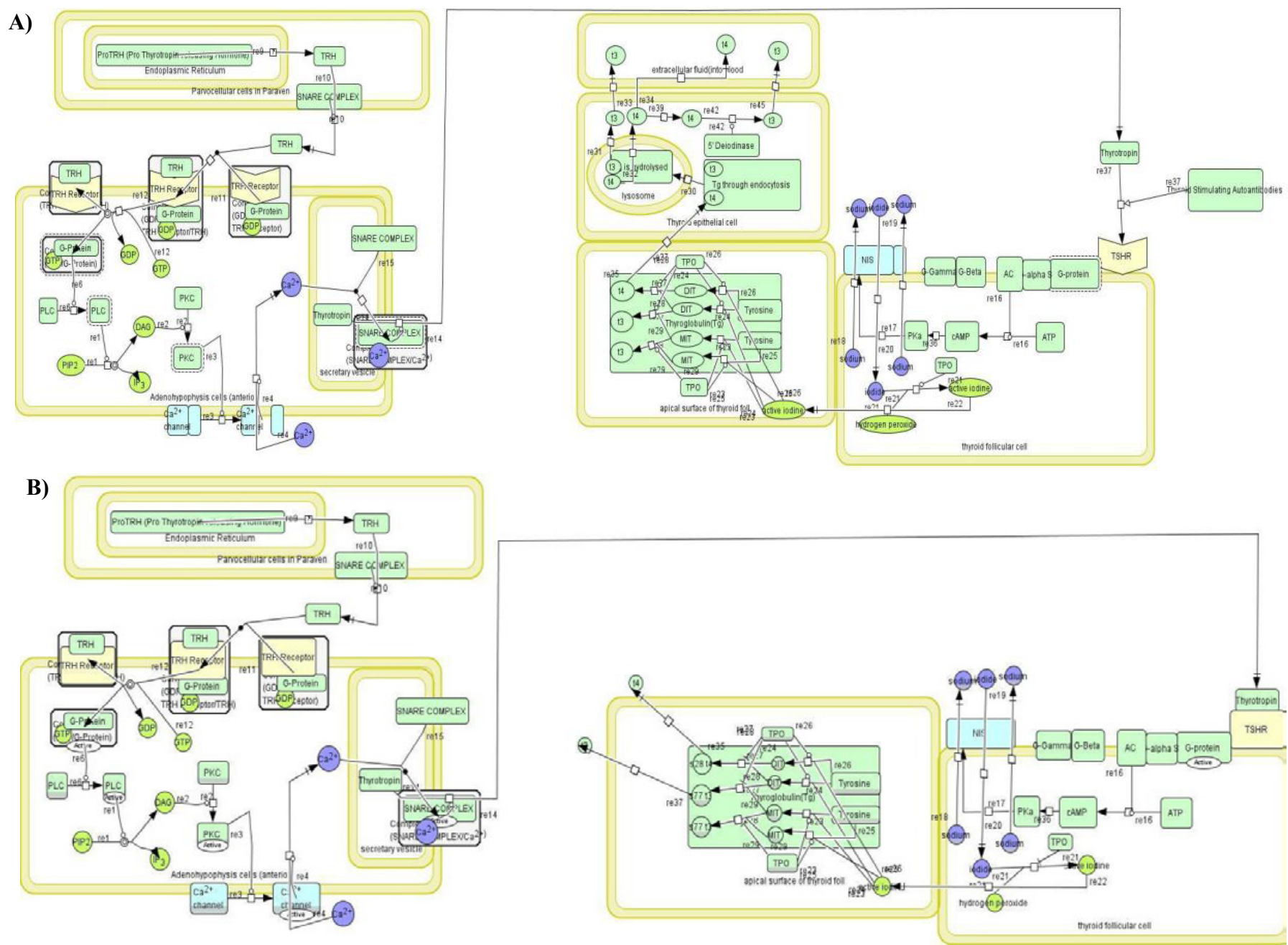

Figure 3: (a) Metabolic pathway of hyper secretion of thyroid hormones of SBGN view. (b) Metabolic pathway of hyposecretion of thyroid hormones SBGN view. 
Citation: Manish Kumar G, Dev Bukhsh S, Rath SK, Krishna M (2012) Metabolic Modeling and Simulation Analysis of Thyroid Disorder Pathway. J Comput Sci Syst Biol 5: 052-061. doi:10.4172/jcsb.1000090

to other publications. Normal level of expertise of a pathway database curator may not be adequate to decide these concerns in an organized and self consistent way. Standardization of pathway knowledge demonstration is of outmost significance in the progression of pathway curation.

\section{Case study}

As an example of the potentialities of the map we performed a study of sixteen cases of both hyperthyroidism and hypothyroidism. From thyroid function tests, the concentration of TSH, T3 and T4 were taken into consideration. These three entities were selected to observe their dynamic behavior by carrying out simulation. In normal condition the reference range of TSH, free T3 and free T4 are $0.34-3.50 \mathrm{mIU} / 1,2.98$ - $5.24 \mathrm{pmol} / \mathrm{L}$ and $9.14-23.8 \mathrm{pmol} / \mathrm{L}$ respectively [10]. Total Sixteen cases were taken for study, out of which 8 different cases related with hypothyroidism and 8 different cases from hyperthyroidism. For simulation analysis of hyperthyroidism which varies from patient to patients in eight different cases, the concentration of TSH , 0.006 to $144 \mathrm{mU} / \mathrm{L}, \mathrm{T} 3,1$ to $35.42 \mathrm{pmol} / \mathrm{L}$ and T4, 5.2 to $90.3 \mathrm{pmol} / \mathrm{L}$ values were taken from published literature. Hypothyroid Graves' disease is a condition when found with TSH - R-AB s and ophthalmic Graves' disease (OGD Cardiac arrhythmias and contractile dysfunction may be the key signs of thyroid storm in young patients was taken into the study [6].

For simulation analysis of metabolic model of hypothyroidism the concentration of TSH varies from 0.005 to $1.78 \mathrm{mU} / \mathrm{L}$, T3 varies from 1.71 to $10.34 \mathrm{pmol} / \mathrm{L}$ and T4 from 10.4 to $16.0 \mathrm{pmol} / \mathrm{L}$. To convert serum free T4 and free T3 values to pmol/L, multiply by 12.9 and 15.4, respectively.

Figure 5 simulation results with different concentration of TSH, free T3 and free T4 (a) normal secretion of thyroid hormones (b)
Simulation result of hypersecretion of thyroid hormones (c) Simulation result of hyposecretion of thyroid hormones. In Figure 6 (a), (b), (c) Simulation was performed using three different initial concentrations of TSH, T3 and T4. In all the three cases, we observed the same type of variation pattern for the three parameters i.e. TSH, T3 and T4. We can say that concentration of TSH, T3 and T4 were increasing with time and after 10 seconds it becomes constant.

Figure 7(a), (b), (c) reveals that by taking initial concentrations of TSH, T3 and T4 for Hypothyroid Grave Disease. Four cases of Hypothyroid Grave Disease were taken for studying the effect of TSH, T3 and T4 in that particular condition. In all the four cases, we observed the same type of variation pattern for the TSH, T3 and T4, although the simulation starts with at different initial condition. In the first two cases, there is rapid fall in the concentration of TSH with the time up to 10 seconds, during the same time period the concentration of T3 and T4 is increasing with time and after 10 seconds it becomes constant. The overall simulation result for Hypothyroid Grave Disease indicates that the concentration of $\mathrm{T} 3$ and $\mathrm{T} 4$ is increasing along with the decrease in the concentration of TSH leading to a diseased condition i.e. Hypothyroid Grave Disease

Figure 8 explain abnormal situation the concentration of TSH is very low as compared to T3 and T4. The T3 and T4 concentration is increasing very fast as compare to TSH, which becomes constant after duration of 10 seconds. This study suggests that higher concentration of T3 and T4 and rapid increase in its level with the time is significant to cause an abnormality (Table 1).

Simulation of Hashimoto Thyroiditis disease Pathway was done by taking unit concentrations (1.0) for TSH, T3 and T4. Simulation result for unit concentration in Hashimoto Thyroiditis is shown in figure 9 (a) and (b). In HT model though, the simulation starts with

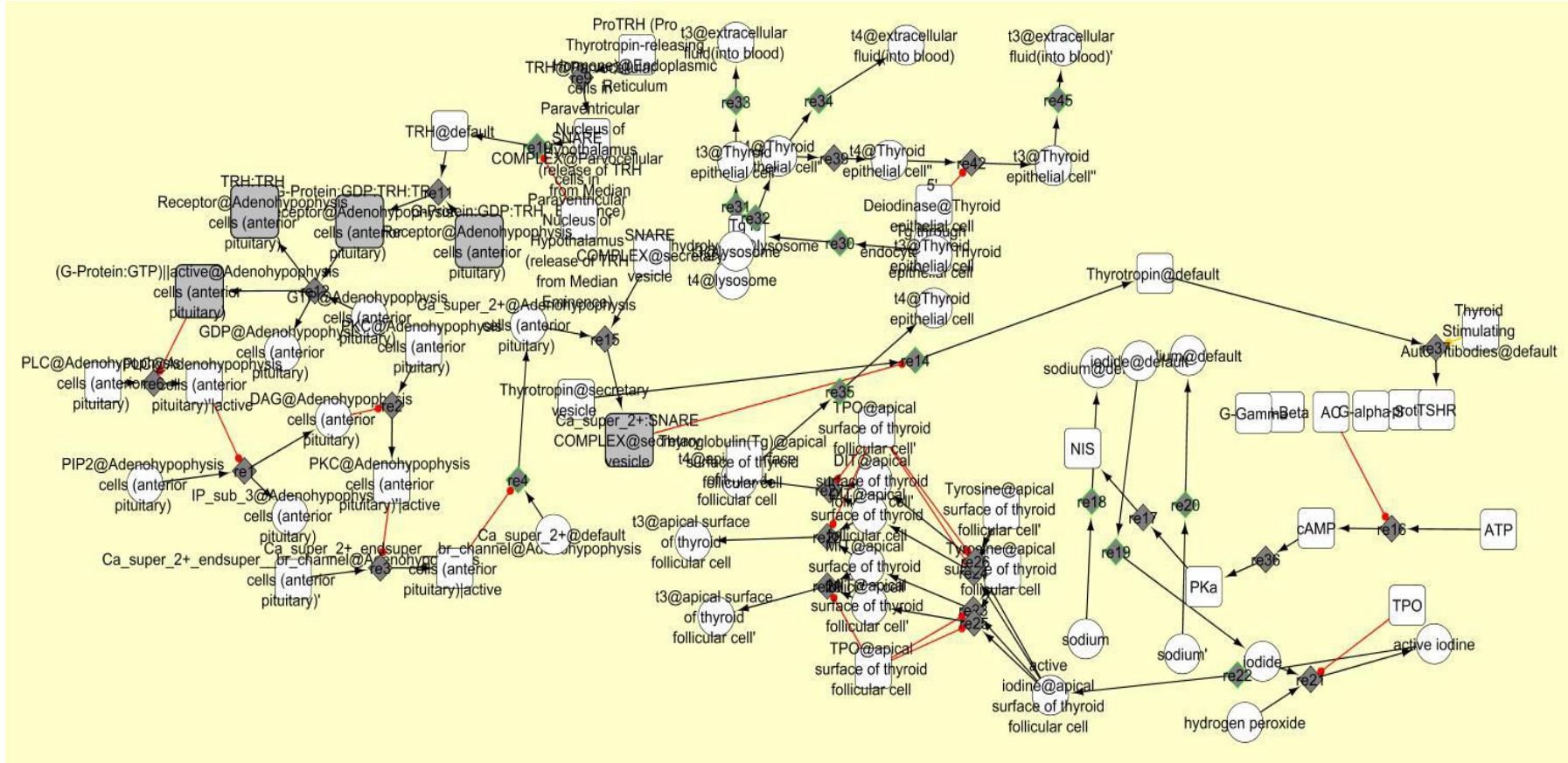

Figure 4: Modular view of Hyperthyroidism network. 
Citation: Manish Kumar G, Dev Bukhsh S, Rath SK, Krishna M (2012) Metabolic Modeling and Simulation Analysis of Thyroid Disorder Pathway. J Comput Sci Syst Biol 5: 052-061. doi:10.4172/jcsb.1000090

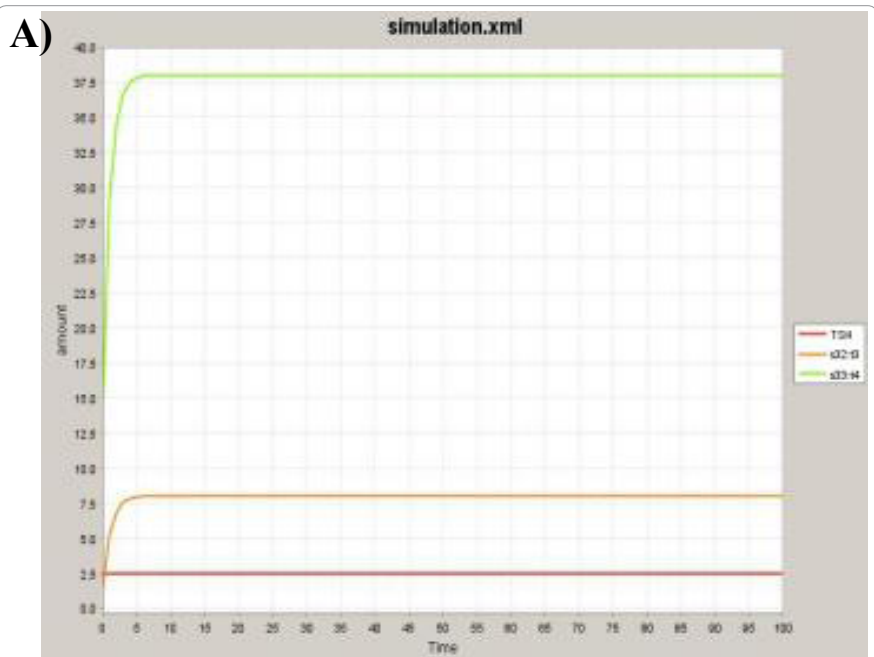

B)

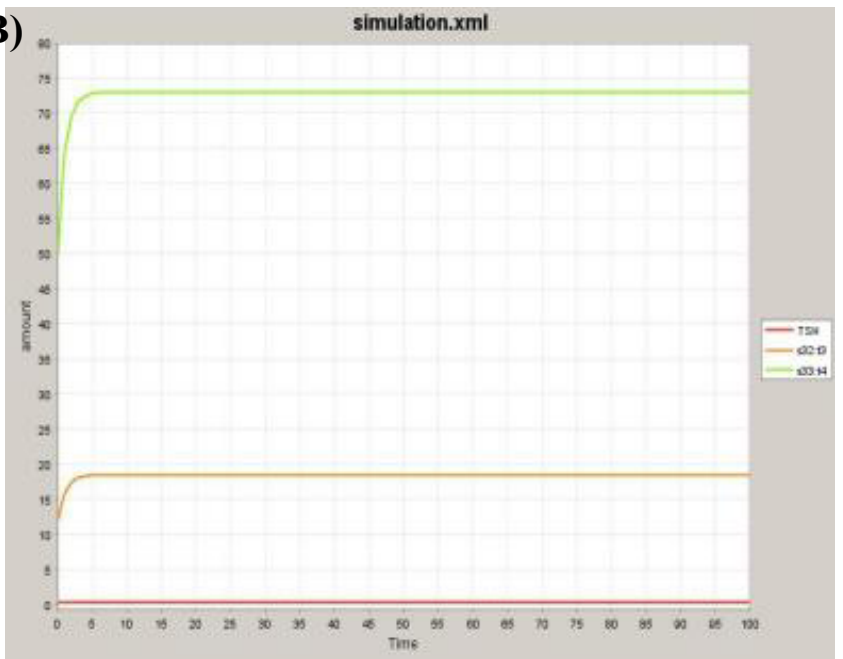

C)

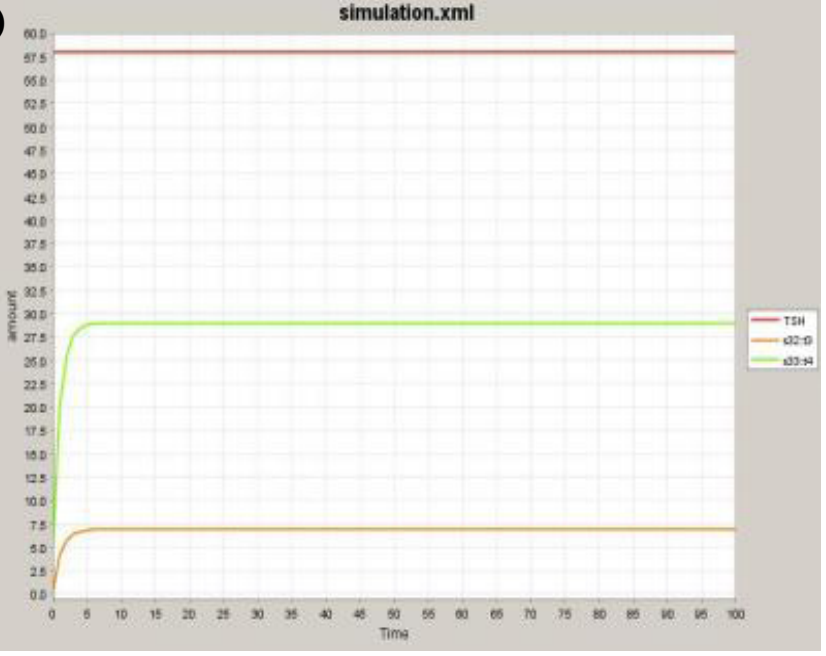

Figure 5: simulation results with different concentration of TSH, free T3 and free T4 (a) normal secretion of thyroid hormones (b) Simulation result of hypersecretion of thyroid hormones (c) Simulation result of hyposecretion of thyroid hormones.

a unit concentration of TSH, T3 and T4, but concentration of T3 and T4 is declining with time as the concentration of TSH is rising. In the six cases from figure 9 (c) to (h), the initial concentration of TSH is

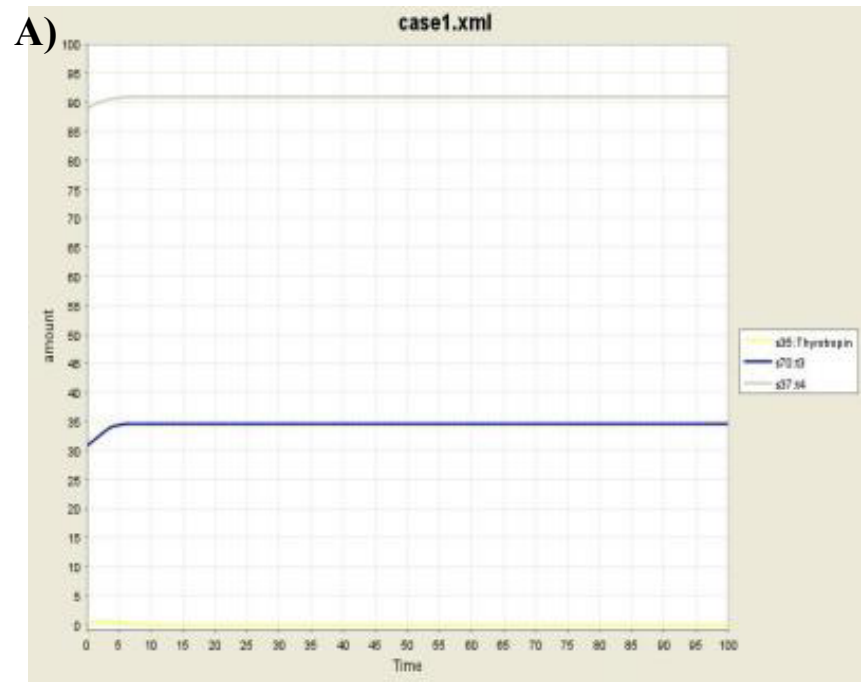

B) case2.xml

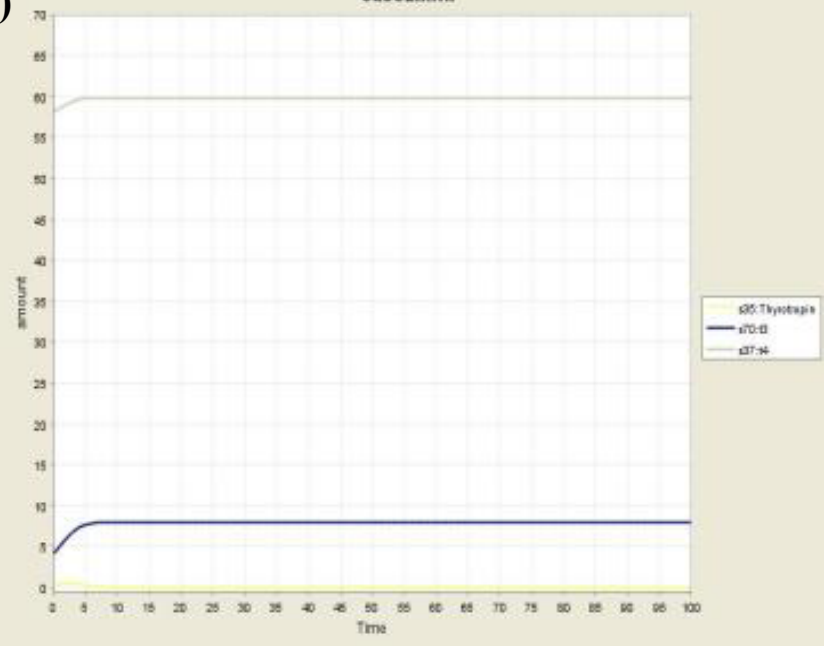

C)

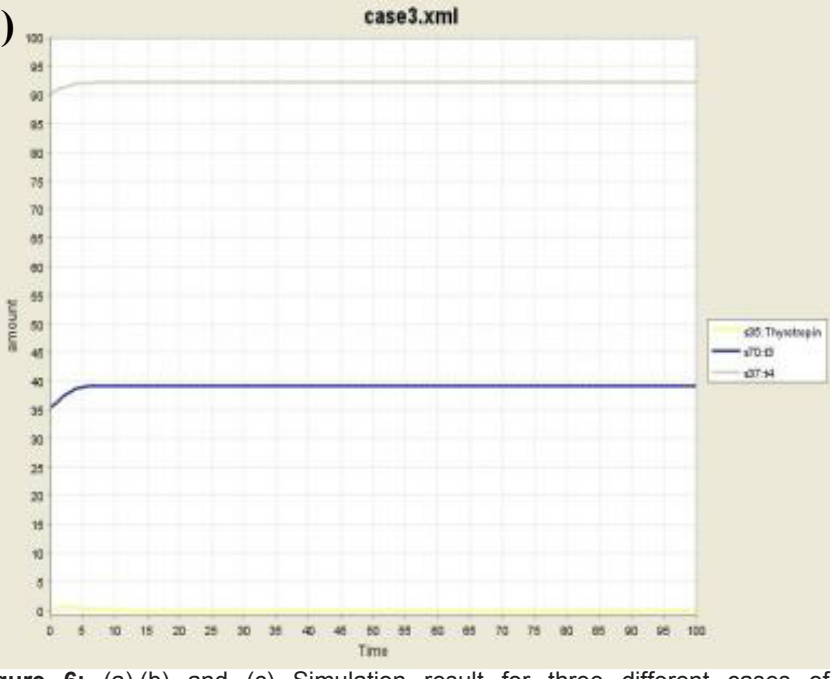

Figure 6: (a),(b) and (c) Simulation result for three different cases of Hyperthyroidism related with cardiac disease, variation in concentrations of $\mathrm{TSH}$ T3 and T4 with the time is shown in yellow, blue and grey color respectively.

In Figure 6 (a), (b), (c) Simulation was performed using three different initial concentrations of TSH, T3 and T4. In all the three cases, we observed the same type of variation pattern for the three parameters i.e. TSH, T3 and T4. We can say that concentration of TSH, T3 and T4 were increasing with time and after 10 seconds it becomes constant. 


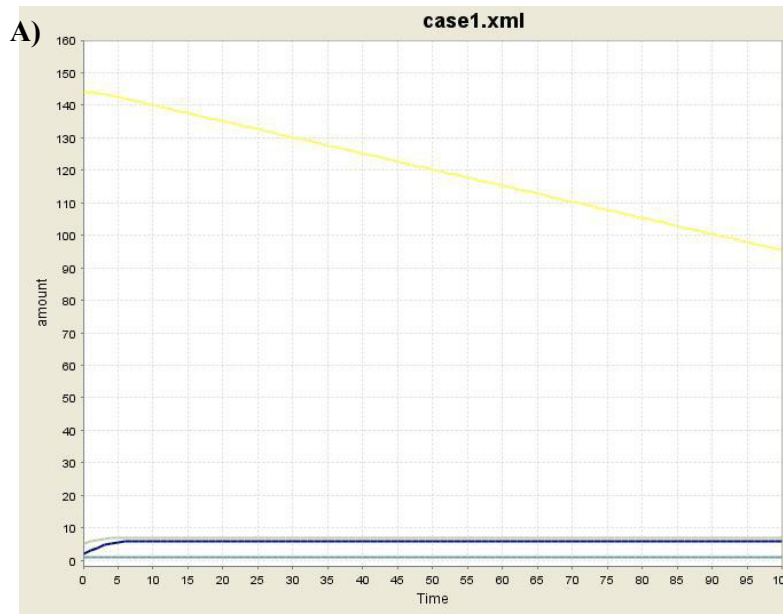

C)

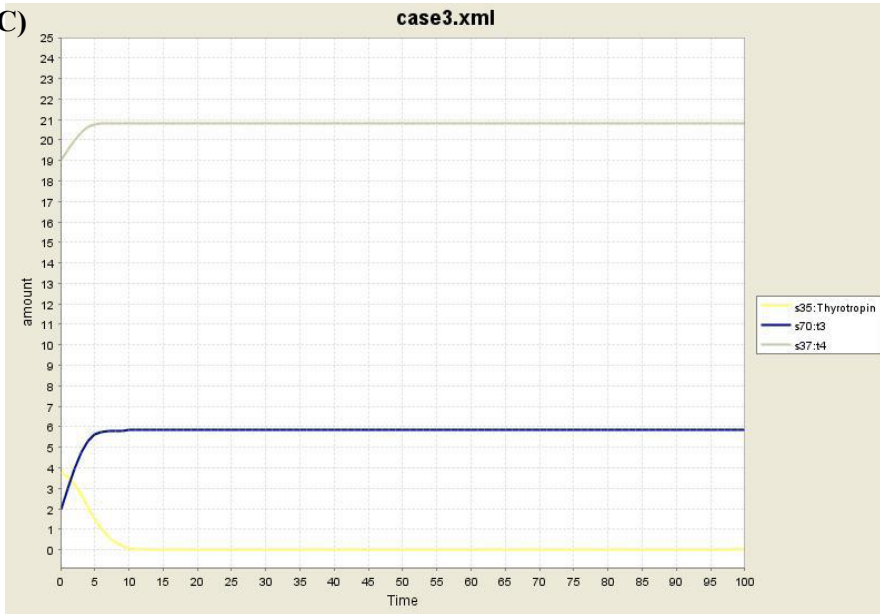

B)

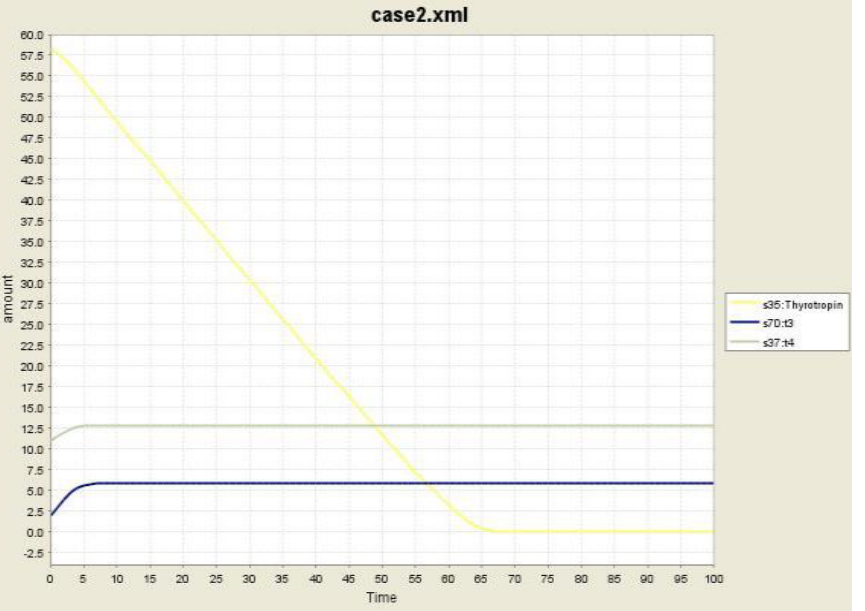

D)

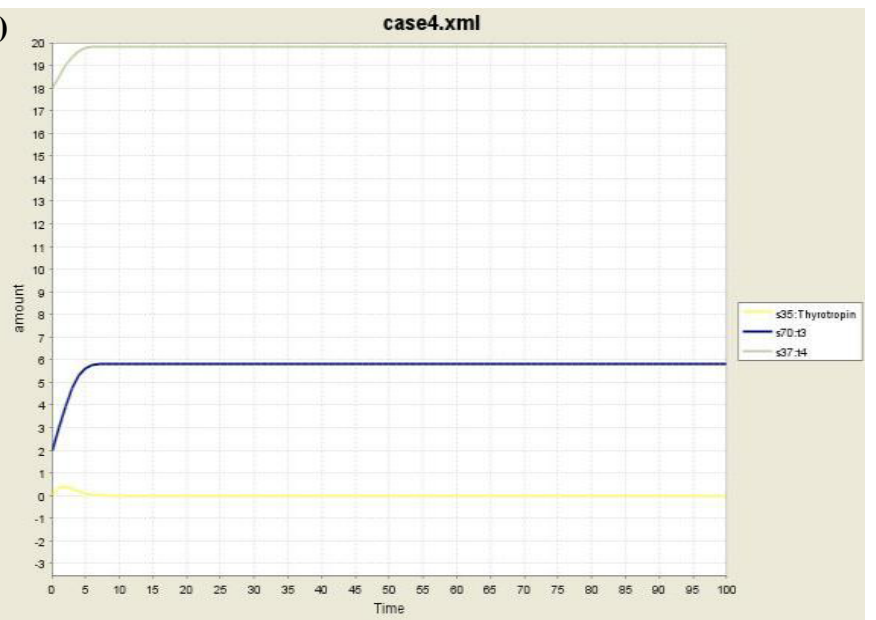

Figure 7: (a), (b), (c) and (d) showing simulation study for Hypothyroid Grave Disease with Four patients, variation in concentrations of TSH, T3 and T4 with the time is shown in yellow, blue and grey color respectively.

much lower than T3 and T4 concentration. The concentration of TSH, $\mathrm{T} 3$ and T4 used for simulation for different cases is given in Table 1. There is significant rise in the concentration of TSH with the decrease in the concentration of $\mathrm{T} 3$ and $\mathrm{T} 4$ with the time. The overall simulation result for Hashimoto Thyroiditis indicates that the concentration of T3 and T4 is falling along with the increase in the concentration of TSH, which finally leads to a diseased condition. These studies reveals that behavior of simulation pattern for concentration of TSH, T3 and T4 is just reverse in case of Hashimoto Thyroiditis as compared to Grave Disease.

Simulation analysis in figure 10 (a) and (b) was based on data from Hashimoto Encephalopathy. The initial conc. of TSH, free T3 and free T4 is given in Table 1. Simulation starts with a lower concentration of TSH and High concentration of T3 and T4. But during the simulation time concentration of T3 and T4 is falling rapidly with the increase in concentration of TSH up to 20 seconds. The pattern of simulation result in Hashimoto Encephalopathy is very much similar to the pattern observed in Hashimoto Thyroiditis.

The simulation was performed for $100 \mathrm{sec}, 1000,10,000$ and 100,000 but the simulation patterns are invariable after passing with certain period, it does not depart the simulation pattern of the pathways. Known biological interactions, networks may be examined with respect to their structural and dynamical patterns, which are associated with phenotypes of interest.

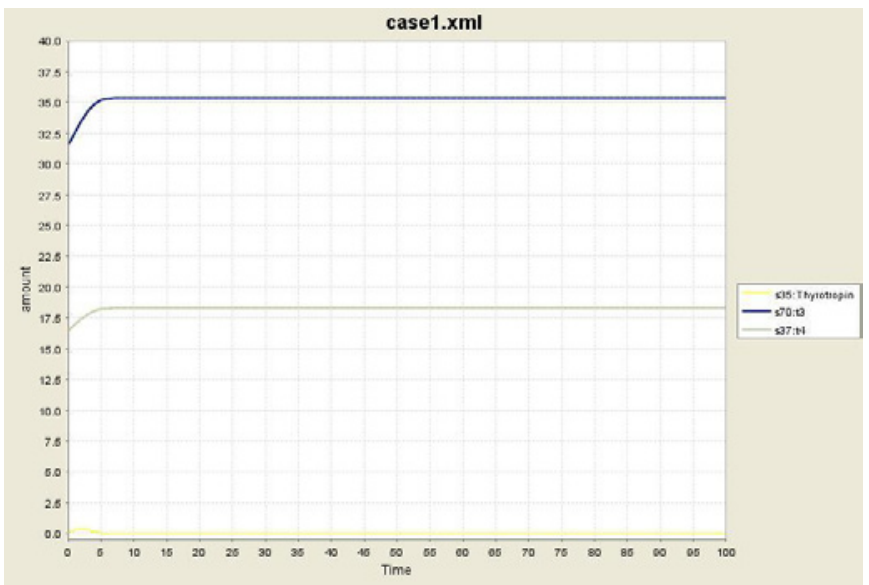

Figure 8: Simulation study for GD presenting patient with pseudo tumour cerebri, variation in concentrations of TSH, T3 and T4 with the time is shown in yellow, blue and grey color respectively. 
Citation: Manish Kumar G, Dev Bukhsh S, Rath SK, Krishna M (2012) Metabolic Modeling and Simulation Analysis of Thyroid Disorder Pathway. J Comput Sci Syst Biol 5: 052-061. doi:10.4172/jcsb.1000090

A) Thyrotropin-releasing_hormone_receptor_signaling_pathway_hasimoto thyroditis.xml

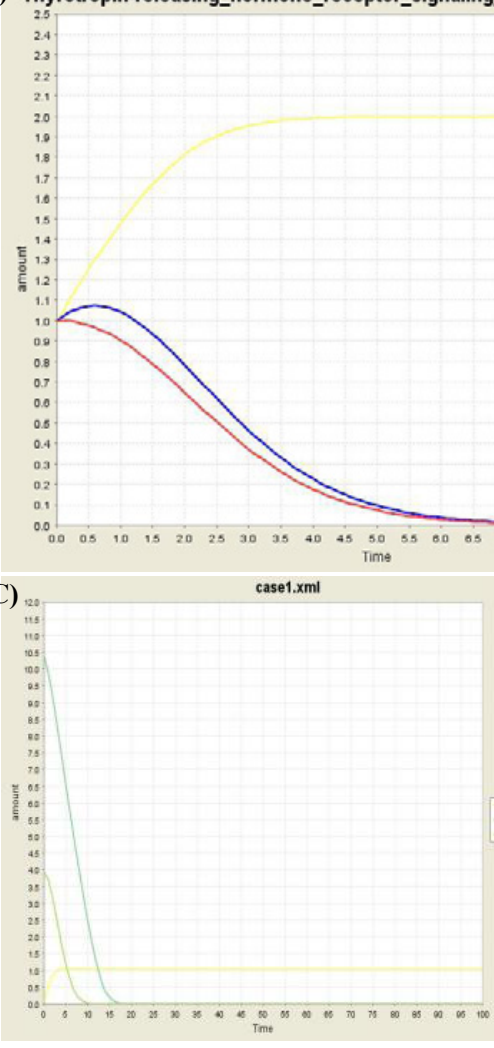

F)

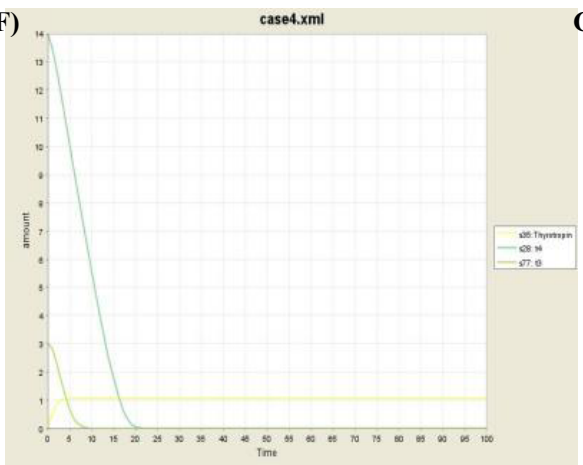

D)

G)
B) Thyrotropin-releasing_hormone_receptor_signaling_pathway_hasimoto thyroditis.xml

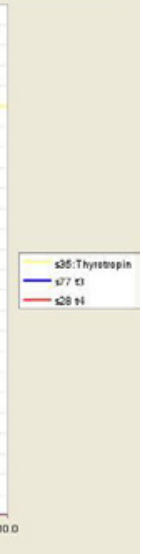

case2.xml
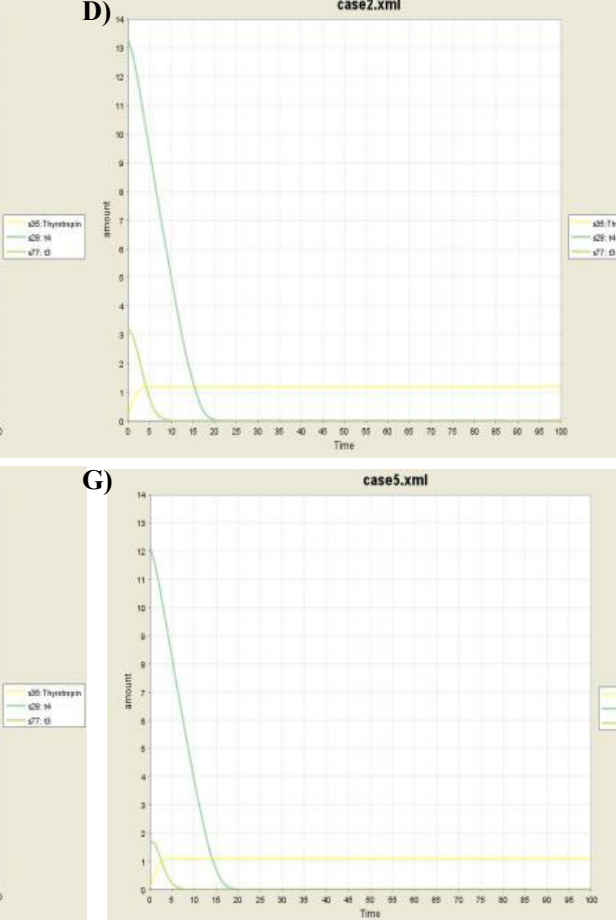

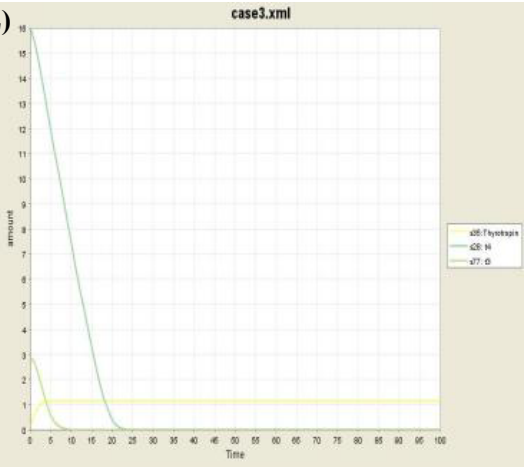

H)

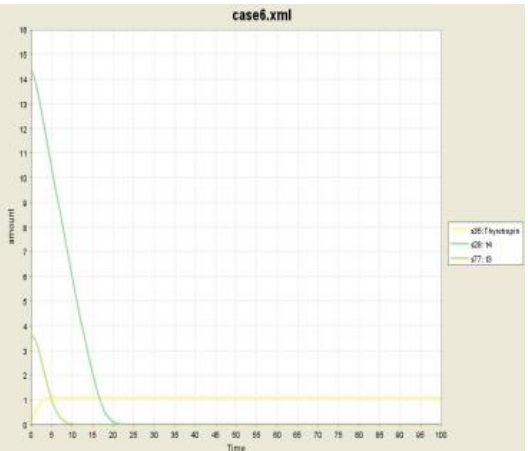

Figure 9: (a) to (h) showing simulation result for Hashimoto Thyroiditis with six patients, variation in concentrations of TSH, T3 and T4 with the time is shown in yellow, light green and dark green colors respectively.
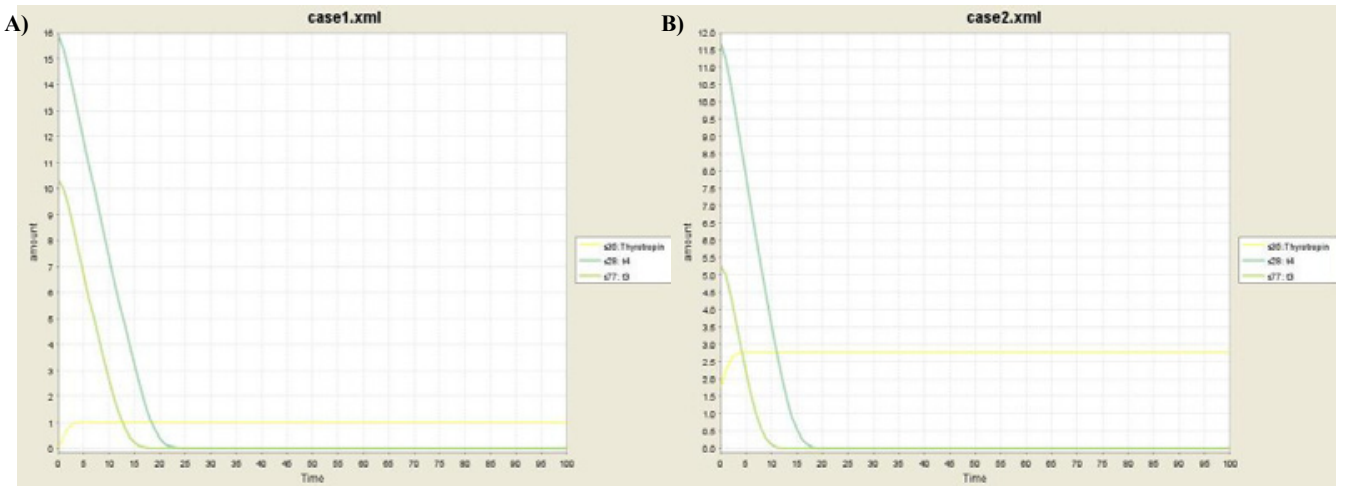

Figure 10: (a) and (b), showing simulation result for two different cases of Hashimoto Encephalopathy Variation in concentrations of TSH, T3 and T4 with the time is shown in yellow, light green and dark green color respectively. 
Citation: Manish Kumar G, Dev Bukhsh S, Rath SK, Krishna M (2012) Metabolic Modeling and Simulation Analysis of Thyroid Disorder Pathway. J Comput Sci Syst Biol 5: 052-061. doi:10.4172/jcsb.1000090

\begin{tabular}{|c|c|c|c|c|c|}
\hline S. No & $\begin{array}{l}\text { Cases with their } \\
\text { Clinical characteristics }\end{array}$ & Age/Sex & TSH (mU/L) & T3 (pmol/L) & T4 (pmol/L) \\
\hline & \multicolumn{5}{|c|}{ Hyperthyroidism } \\
\hline \multicolumn{6}{|c|}{ Hypothyroid Graves Disease [6] } \\
\hline 1. & Case1: Fatigue, unilateral lid retraction and hypertension & $60 / F$ & 144 & ND & 5.2 \\
\hline 2. & $\begin{array}{l}\text { Case2: Fatigue and weight gain, mild periorbital oedema, unilateral lid } \\
\text { retraction and exophthalmos of the right eye }\end{array}$ & $44 / \mathrm{M}$ & 58.2 & ND & 11 \\
\hline 3. & $\begin{array}{l}\text { Case3: Severe congestive Graves' } \\
\text { ophthalmopathy and signs of optic nerve involvement }\end{array}$ & $69 / F$ & 3.8 & ND & 19 \\
\hline 4. & Case4: Exophthalmos and subjective Pressure sensations behind eyes & $44 / F$ & 0.023 & ND & 18 \\
\hline \multicolumn{6}{|c|}{ Graves' disease with pseudotumor cerebri [7] } \\
\hline 5. & Case1: Persistent headache, vomiting and nausea & $31 / F$ & 0.01 & 31.6 & ND \\
\hline \multicolumn{6}{|c|}{ Disorder Related with cardiac disease [8] } \\
\hline 6. & Case1: Dyspnea, edema, tremor, and heat intolerance & $32 / \mathrm{M}$ & $<0.006$ & 30.8 & 89.01 \\
\hline 7. & Case2: Dyspnea, orthopnea, and anasarca & 28/M & $<0.006$ & ND & 58.05 \\
\hline \multirow[t]{2}{*}{8.} & Case3: Anorexia, weight loss, diarrhea, and heat intolerance & $30 / \mathrm{M}$ & $<0.01$ & $>35.42$ & $>90.3$ \\
\hline & \multicolumn{5}{|c|}{ Hypothyroidism } \\
\hline \multicolumn{6}{|c|}{ Hashimoto's encephalopathy [9] } \\
\hline 9. & $\begin{array}{l}\text { Case: } 1 \text { Headache localised in the frontal and occipitoparietal regions, } \\
\text { nausea, vomiting and slightly altered hearing }\end{array}$ & $29 / F$ & 0.005 & 10.34 & 15.89 \\
\hline 10. & Case2: Transient mental confusion & $65 / F$ & 1.78 & 5.28 & 11.71 \\
\hline \multicolumn{6}{|c|}{ Diagnosis of isolated deficit of TSH [10] } \\
\hline 11. & Case1: Cold intolerance, leg heaviness, . Sleepiness, constipation & $58 / F$ & $<0.04$ & 3.94 & 10.4 \\
\hline 12. & Case2: Fatigability, leg heaviness & 23/F & 0.19 & 3.18 & 13.3 \\
\hline 13. & Case3: Fatigability & $20 / F$ & 0.13 & 2.87 & 16.0 \\
\hline 14. & Case4: Fatigability, Sleepiness, constipation & $52 / \mathrm{M}$ & 0.05 & 3.01 & 14.0 \\
\hline 15. & Case5: Lethargy, constipation & $64 / F$ & 0.10 & 1.71 & 12.1 \\
\hline 16. & Case6: Fatigability, constipation & $60 / F$ & 0.06 & 3.64 & 14.4 \\
\hline
\end{tabular}

Table 1: The quantitative level of TSH, T3 and T4 in different cases of thyroid disease.

\section{Conclusion}

The goal of this study is to map and understanding the complex reactions which takes place in human body during thyroid hormone secretion, in hyperthyroidism and hypothyroidism. This study can help in finding the novel drug target. As these pathways has been designed for understanding the underlying complexity in the disease state and normal state with the time dependent behavior of different entities. By measuring the hormonal level of patients and performing the simulation using these data can lead to better understanding of the fluctuations taking place in the hormonal concentrations with respect to time. By observing the graph, it can be concluded that the concentration of TSH remains constant at a particular level and concentrations of T3, T4 varies with time up to a certain level and then becomes constant in both Hyperthyroidism and Hypothyroidism. TSAb and TBAb binds with TSHR and either mimic or block the action of TSH resulting in hyperthyroidism or hypothyroidism. So, TSHR can be used as a drug target. Thus, a drug which can bring the level of TSH, $\mathrm{T} 3$ and T4 to reference range of concentration can act as potential drug. To anticipate potential drug targets by system-wide analysis of the metabolic network for the effective treatment of thyroid disorder, the model can be useful.

In silico biology has an advantage over experimental biology in context to expenditure, smoothness and speediness. The system Modeling can provide an important insight into the operational and common principles of organization of biological systems. Also it can propose new experiments for testing assumption, based on the modeling practice.

\section{References}

1. Swain M, Swain T, Mohanty BK (2005) Auto-immune thyroid disorders-an update. Indian J Clinic Bio chem 20: 9-17.

2. Chazenbalk GD, Pichurin P, Chen CR, Latrofa F, Johnstone AP, et al. (2002) Thyroid-stimulating autoantibodies in Graves disease preferentially recognize the free A subunit, not the thyrotropin holoreceptor. J Clin Invest 110: 209-217.

3. Cho BY (2002) Clinical applications of TSH receptor antibodies in thyroid diseases. J Korean Med Sci 17: 293-301.

4. Morgenthaler NG, Ho SC, Minich WB (2007) Stimulating and blocking thyroidstimulating hormone (TSH) receptor autoantibodies from patients with Graves disease and autoimmune hypothyroidism have very similar concentration, TSH receptor affinity, and binding sites. J Clin Endocrinol Metab 92: 1058-1065.

5. Chen CR, Tanaka K, Chazenbalk GD, McLachlan SM, Rapoport B (2001) A ful biological response to autoantibodies in Graves' disease requires a disulfidebonded loop in the thyrotropin receptor $\mathrm{N}$ terminus homologous to a laminin epidermal growth factor-like domain. J Biol Chem 276: 14767-14772.

6. Starrenburg-Razenberg AJ, Castro Cabezas M, Gan IM, Njo TL, Rietveld AP et al. (2010) Four patients with hypothyroid Graves' disease. Neth J Med 68: 178-180.

7. Coutinho E, Silva AM, Freitas C, Santos E (2011) Graves' disease presenting as pseudotumor cerebri: a case report. J Med Case Reports 5:68.

8. Ngo SY, Chew HC (2007) Cardiac abnormalities may be the dominant manifestations of thyroid storm in young men. Clinical Thyroidology 19: 43.

9. Tamagno G, Federspil G, Murialdo G (2006) Clinical and diagnostic aspects of encephalopathy associated with autoimmune thyroid disease (or Hashimoto's encephalopathy). Intern Emerg Med 1: 15-23.

10. Yamakita N, Komaki T, Takao T, Murai T, Hashimoto K, et al. (2001) Usefulness of thyrotropin (TSH)-releasing hormone test and nocturnal surge of TSH for diagnosis of isolated deficit of TSH secretion. J Clin Endocrinol Metab 86 1054-1060. 
Citation: Manish Kumar G, Dev Bukhsh S, Rath SK, Krishna M (2012) Metabolic Modeling and Simulation Analysis of Thyroid Disorder Pathway. J Comput Sci Syst Biol 5: 052-061. doi:10.4172/jcsb.1000090

11. Materi W, Wishart DS (2007) Computational systems biology in drug discovery and development: methods and applications. Drug Discov Today 12: 295-303.

12. O'Malley MA, Dupré J (2005) Fundamental issues in systems biology. Bioessays 27: 1270-1276.

13. Butcher EC, Berg EL, Kunkel EJ (2004) Systems biology in drug discovery. Na Biotechnol 22: 1253-1259.

14. Kell DB (2006) Systems biology, metabolic modelling and metabolomics in drug discovery and development. Drug Discov Today 11:1085-1092.

15. Hucka M, Finney A, Sauro HM, Bolouri H, Doyle JC, et al. (2003) The Systems Biology Markup Language (SBML): a medium for representation and exchange of biochemical network models. Bioinformatics 19: 524-531.

16. Kitano H, Funahashi A, Matsuoka Y, Oda K (2005) Using process diagrams for the graphical representation of biological networks. Nat Biotechnol 23: 961-966.

17. Kitano H (2003) A graphical notation for biochemical networks. Biosilico 1: 169176

18. Huaiyu Mi, Betty Lazareva-Ulitsky, Rozina Loo, Anish Kejariwal, Jody Vandergriff, et al. (2005) The PANTHER database of protein families, subfamilies, functions and pathways. Nucl Acids Res. 33: 284-288.

19. Hucka M, Finney A, Bornstein BJ, Keating SM, Shapiro BE, et al. (2004) Evolving a lingua franca and associated software infrastructure for computational systems biology: the Systems Biology Markup Language (SBML) project. Syst Biol (Stevenage) 1: 41-53.

20. Funahashi A, Tanimura N, Morohashi M, Kitano H (2003) Cell Designer: a process diagram editor for gene-regulatory and biochemical networks. Biosilico 1:159-162.

21. Funahashi A, Jouraku A, Matsuoka $Y$, Kitano $H$ (2007) Integration of CellDesigner and SABIO-RK. In Silico Biol 7: S81-90.

22. Funahashi A, Matsuoka Y, Jouraku A, Morohashi M, Kikuchi N, et al. (2008) CellDesigner 3.5: A Versatile Modeling Tool for Biochemical Networks. Proceedings of the IEEE 96: 1254 - 1265.

23. Dräger A, Hassis N, Supper J, Schröder A, Zell A (2008) SBMLsqueezer: a CellDesigner plug-in to generate kinetic rate equations for biochemical networks. BMC Syst Biol 2:39.
24. Zinovyev A, Viara E, Calzone L, Barillot E (2008) BiNoM: a Cytoscape plugin for manipulating and analyzing biological networks. Bioinformatics 24: 876-877.

25. Shannon P, Markiel A, Ozier O, Baliga NS, Wang JT, et al. (2003) Cytoscape: a software environment for integrated models of biomolecular interaction networks. Genome Res 13: 2498-2504.

26. Kohl M, Wiese S, Warscheid B (2011) Cytoscape: software for visualization and analysis of biological networks. Methods Mol Biol 696: 291-303.

27. Smoot ME, Ono K, Ruscheinski J, Wang PL, Ideker T (2011) Cytoscape 2.8 new features for data integration and network visualization. Bioinformatics 27 : 431-432.

28. Mi H, Muruganujan A, Demir E, Matsuoka Y, Funahashi A, et al. (2011) BioPAX support in CellDesigner. Bioinformatics 27: 3437-3438.

29. Davies TF, Ando T, Lin RY, Tomer Y, Latif R (2005) Thyrotropin receptorassociated diseases: from adenomata to Graves disease. J Clin Invest 115 1972-1983.

30. Davies T, Marians R, Latif R (2002) The TSH receptor reveals itself. J Clin Invest. 110: 161-164

31. Collison KS, Banga JP, Barnett PS, Huang GC, McGregor AM (1991) Autoantibody stimulation of the human thyrotropin receptor: regulation of adenylate cyclase activity, thyroglobulin and thyroid peroxidase mRNA levels in primary cultures of Graves' thyroid tissue. Clin Exp Immunol 86: 61-65.

32. Ajjan RA, Findlay C, Metcalfe RA, Watson PF, Crisp M, et al. (1998) The modulation of the human sodium iodide symporter activity by Graves' disease sera. J Clin Endocrinol Metab 83: 1217-1221.

33. Riesco-Eizaguirre G, Santisteban $P$ (2006) A perspective view of sodium iodide symporter research and its clinical implications. Eur J Endocrinol 155: 495-512.

34. Pathophysiology of the Endocrine System (2006).

35. Weetman AP, McGregor AM (1994) Autoimmune thyroid disease: further developments in our understanding. Endocr Rev 15: 788-830.

36. Bresson D, Rebuffat SA, Péraldi-Roux S (2005) Localization of the immunodominant region on human thyroid peroxidase in autoimmune thyroid diseases: an update. J Autoimmune Dis 2: 2 